\title{
New Trends in Digital Humanities
}

\author{
Roberto Theron ${ }^{1}$ and Eveline Wandl-Vogt ${ }^{2}$ \\ ${ }^{1}$ Department of Computer Science and Automation, University of Salamanca, Salamanca, Spain, email: \\ theron@usal.es \\ ${ }^{2}$ Austrian Academy of Sciences, Austrian Centre for Digital Humanities, Vienna, Austria, email: \\ eveline.wandl-vogt@oeaw.ac.at
}

\begin{abstract}
The field of research in digital humanities is undergoing a rapid transformation in recent years. A deep reflection on the current needs of the agents involved that takes into account key issues such as the inclusion of citizens in the creation and consumption of the cultural resources offered, the volume and complexity of datasets, available infrastructures, etcetera, is necessary. Present technologies make it possible to achieve projects that were impossible until recently, but the field is currently facing the challenge of proposing frameworks and systems to generalize and reproduce these proposals in other knowledge domains with similar but heterogeneous data sets. The track "New trends in digital humanities" of the Fourth International Conference on Technological Ecosystems for Enhancing Multiculturality (TEEM 2016), tries to set the basis of good practice in digital humanities by reflecting on models, technologies and methods to carry the transformation out.
\end{abstract}

\section{Introduction}

Digital Humanities as a research field has a long history if we look for projects that can be placed at the intersection of computing and the disciplines of the humanities. However, it is now with the pervasiveness of computers and a great variety of electronic devices connected to the Internet, that most of the promises of the field can be pursued with sufficient guarantee of success.

The road to follow is not without obstacles. A number of challenges must be faced in order to complete any of the said projects. From the creation to the consumption of digital resources, there are new stakeholders, contexts and tasks to consider.

The role of citizens has changed enormously, for instance, since now we live in a society that, at least theoretically, has democratized science, and the number of projects in which the contribution of citizens, either producing or using digital resources, has exploded.

The experience gained in other areas of science in which the intervention of computing has been much deeper and constant must be analysed and adapted to the case of humanities. Specially, regarding infrastructures, frameworks, models and tools that can be standardized for the different disciplines in the humanities to fully exploit the available technology.

The amount of digital resources produced (or digitized), stored, explored, and analysed in any Digital Humanities project is immensely vast (specially if we take into account the introduction of linked data), so the traditional humanities tools have to be either substituted or aided with ancillary tools in the form of interactive visualisations or novel user interfaces.

Another challenge for the success of any Digital Humanities project is that of a profound understanding of the actual needs of the people involved in the project. Approaching the design of the desired system from a human-computer interaction point of view can pave the way for the adoption of novel proposals in the field.

Finally, some decades after the introduction of the first curricula in Digital Humanities, it is also a moment to reflect on how these courses are preparing students for the future practice of Digital Humanities, asking whether they are acquiring the relevant skills: programming, methodological, multidisciplinary-team working, communication, and so forth.

The track on New trends in Digital Humanities of the Fourth International Conference on Technological Ecosystems for Enhancing Multiculturality (TEEM 2016), tries to set the basis of good practice in digital humanities by reflecting on models, technologies and methods to carry the transformation out. 


\section{Lines of work}

The track is intended to examine the state of the art of Digital Humanities regarding the following topics (and related topics present in other tracks):

- humanities research enabled through digital media, and computational methods

- computer applications in literary, linguistic, cultural, and historical studies

- visual approaches enabling novel ways of analysis and exploration of resources

- studies regarding the role of art and design (design thinking) in humanities

- open science and citizen science enabling cultural change towards collaboration and openness

- enhancing education in humanities through serious games

- digital arts, architecture, music, film, theatre, new media, digital games, and related areas

- creation and curation of humanities digital resources

- social, institutional, global, multilingual, and multicultural aspects of digital humanities

- interdisciplinary aspects of digitally enabled scholarship

- innovative methods of application of 3D, robotics, drones, natural user interfaces, etc. in the context of the humanities

Particularly, the track will trigger open discussion on the above topics that will be organized in four lines of work:

- 1. Open science and Citizen science. Digital humanities is embracing open science and citizen science through an increasing number of projects. However, the experience accumulated is still quite shallow as compared with other scientific fields $[4,5]$. The way data collection, data management, quality control, human-computer interaction and communication, user engagement, etcetera, are carried out will be paramount for the success of these kind of projects.

- 2. Cultural analytics, visualization and innovative user interfaces. The ubiquity of computers and diverse electronic devices and computer networks gave way to an exponential growth in the data available for researching and understanding cultural fenomena. The systematic use of large-scale computational analysis and interactive visualization tools will play a key role in the field of digital humanities $[3,8,7,6]$

- 4. Curriculum design. "Most humanities curricula have a long way to go towards an integration of new computational methods, for a variety of reasons" [2]. This statement from 1999 is still valid [9], and which are the most important skills students in Digital Humanities need to learn are still open to discussion.

- 3. Frameworks, models and infrastructures. Digital collections are proliferating, but most remain difficult to use. Furthermore, Information- and data-intensive, distributed, collaborative, and multidisciplinary research is now the norm in the sciences, while remaining experimental in the humanities [1]. Infrastructure requirements must be fully understood in order to leverage frameworks and models that permit to advance in the field.

\section{Accepted papers}

Six papers have been accepted in this track, with one paper in line 1 (Open science and citizen science), one paper in line 2 (Cultural analytics, visualization and innovative user interfaces), and two papers in lines 3 (Curriculum design) and 4 Frameworks, models and infrastructures.

Papers in line 1 (Open science and citizen science): 
- What is translational literature and how to classify it? Crowd-sourcing as a starting point for corpus building and type distinction in Comparative Literature. The authors propose a collaborative crowd-sourcing project based on litblogs, designed to inspire both the search for relevant texts as well as determining their categorization.

Papers in line 2 (Cultural analytics, visualization and innovative user interfaces):

- A spatio-temporal visual analysis tool for historical dictionaries. This paper presents a tool aimed at improving the comprehension of a massive amount of data (a rich and unique collection of words of the Bavarian dialects recorded during the former Austrian-Hungarian Monarchy period and beyond) through visualization means, thus trying to help in the reach of meaningful conclusions and the acquisition of valuable insights in an easy and fast way.

Papers in line 3 (Frameworks, models and infrastructures):

- From syllables, lines and stanzas to linked open data: standardization, interoperability and multilingual challenges for digital humanities. This proposal presents the challenges and first results of POSTDATA ERC Starting Grant project, which aims at bridging the digital gap among traditional poetry collections and the growing world of data. It is focused on poetry analysis, classification and publication, and applying Digital Humanities methods of academic analysis in order to look for standardization.

- Extracting static and dynamic model elements from textual specifications in humanities. This paper proposes a pipeline aimed at reducing the domain understanding complexity required to software analysts in humanities information system conception and improving the quality of the models in both static and dynamic dimensions.

Papers in line 4 (Curriculum design):

- New insights into translation-oriented, technology intensive localiser education: accessibility as an opportunity. This paper approaches both the practice and the teaching of localisation, incorporating accessibility as a key factor.

- Engaging Students in Curriculum Development: The Case of an Undergraduate Course in Digital Humanities. An innovative, participatory curriculum development project for Digital Humanities for Spanish Graduate Students is presented, which involves undergraduate students as consultants and co-producers of course content.

\section{Conclusions}

Current challenges in Digital Humanities can only be faced by means of well-established and widelyknown good practices. The field must take advantage of the experience gained in other scientific fields that incorporated open science, citizen science, visualization and appropriate standardized frameworks, models and infrastructures. The actual set of skills that students and practitioners of Digital Humanities must be expanded and updated to consider the needs of the present sociocultural context.

This track will contribute with the dissemination of some good practices, as well as with the open discussion of the current challenges of the field both among the participants in this track, but also among the participants of other tracks that share similar problems.

\section{Acknowledgments}

The chairs of this track for Educational Innovation wish to acknowledge and thank the hard work of the Scientific Committee for this track. They reviewed, recommended and evaluated the submitted papers. We also wish to thank the organising committee of Teem 16 for their great coordination, management and integration of all the tracks; thanks to them the organisation of this track has been made possible.

Finally, the authors would like to express their gratitude to the GRIAL research group (http://grial.usal.es). 


\section{References}

[1] C. L. Borgman. The digital future is now: A call to action for the humanities. Digital humanities quarterly, 3(4), 2009.

[2] K. de Smedt, H. Gardiner, E. Ore, T. Orlandi, H. Short, J. Souillot, and W. Vaughan. Computing in humanities education. A European Perspective. SOCRATES/ERASMUS thematic network project on Advanced Computing in the Humanities. University of Bergen, 1999.

[3] L. Manovich. Cultural analytics: visualising cultural patterns in the era of "more media". Domus March, 2009.

[4] G. Newman, A. Wiggins, A. Crall, E. Graham, S. Newman, and K. Crowston. The future of citizen science: emerging technologies and shifting paradigms. Frontiers in Ecology and the Environment, 10(6):298-304, 2012.

[5] J. Silvertown. A new dawn for citizen science. Trends in ecology $\mathscr{E}$ evolution, 24(9):467-471, 2009.

[6] R. Theron and L. Fontanillo. Diachronic-information visualization in historical dictionaries. Information Visualization, 14(2):111-136, 2015.

[7] R. Therón, C. Seguín, L. De la Cruz, and M. Vaquero. Highly interactive and natural user interfaces: Enabling visual analysis in historical lexicography. In Proceedings of the First International Conference on Digital Access to Textual Cultural Heritage, pages 153-158. ACM, 2014.

[8] R. Therón Sánchez and E. Wandl-Vogt. The fun of exploration: How to access a non-standard language corpus visually. 2014.

[9] D. Zorich. A survey of digital humanities centers in the United States. Council on Library and Information Resources Washington, DC, 2008. 